\title{
DÉVELOPPEMENTS EN SÉRIE DANS UNE AIRE \\ LIMITEE PAR DES ARCS DE CERCLE
}

PAR

P. APPELL

à PARIS.

Exemple 1 .

Considérons quatre cercles ayant pour rayon $\frac{1}{\sqrt{2}}$ et pour centres les points

$$
+1,+i,-1,-i \text {. }
$$

L'espace situé à l'extérieur de ces quatre cercles est composé de deux parties

$\left.1^{\circ}\right)$ Une aire finie $A B C D$ qui contient lorigine $O$.

$\left.2^{\circ}\right)$ Une aire indéfinie.

Je vais former par la méthode générale indiquée dans les Comptes Rendus( $\left(^{1}\right)$ une série de fractions rationnelles qui converge dans ces deux aires et qui,

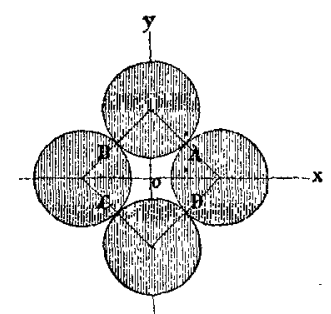
dans l'aire finie $A B C D$, a pour somme 1 et dans l'aire indéfinie est égale à 0 . D'après la méthode générale ce développement sera de la forme

$$
1=\sum_{n=1}^{n=\infty}\left[\frac{A_{n}^{(1)}}{(x-1)^{n}}+\frac{A_{n}^{(i)}}{(x-i)^{n}}+\frac{A_{n}^{(-1)}}{(x+1)^{n}}+\frac{A_{n}^{(-i)}}{(x+i)^{n}}\right] .
$$

Les coefficients de ce développement sont donnés par les formules suivantes:

$$
A_{n}^{(1)}=-\frac{1}{2 \pi i} \int_{D A}(z-1)^{n-1} d z
$$

(') Séance du premier Mai 1882.

Acta mathematica. I. 
l'indice $D A$ indiquant que l'intégration est faite sur l'arc de cercle $D A$. Si l'on pose

$$
z=1+\frac{1}{\sqrt{2}} e^{\theta i}
$$

il faudra faire varier $\theta$ de $-\frac{3 \pi}{4} \grave{\mathrm{a}}-\frac{5 \pi}{4}$. Donc

On trouve de mème

$$
A_{n}^{(1)}=-\frac{1}{2 \pi i} \cdot \frac{1}{(\sqrt{2})^{n}} \int_{-\frac{3 \pi}{4}}^{-\frac{5 \pi}{4}} e^{n A i} i d \theta=\frac{(-1)^{n}}{\pi n(\sqrt{2})^{n}} \cdot \operatorname{Sin} \frac{n \pi}{4}
$$

$$
\begin{aligned}
& A_{n}^{(i)}=-\frac{1}{2 \pi i} \int_{A B}(z-i)^{n-1} d z=\frac{(-i)^{n}}{\pi n(\sqrt{\overline{2}})^{n}} \cdot \operatorname{Sin} \frac{n \pi}{4} \\
& A_{n}^{(-1)}=-\frac{1}{2 \pi i} \int_{B C}(z+1)^{n-1} d z=\frac{1}{\pi n(\sqrt{2})^{n}} \cdot \operatorname{Sin} \frac{n \pi}{4} \\
& A_{n}^{(-i)}=-\frac{1}{2 \pi i} \int_{C D}(z+i)^{n-1} d z=\frac{i^{n}}{\pi n(\sqrt{2})^{n}} \cdot \operatorname{Sin} \frac{n \pi}{4}
\end{aligned}
$$

En portant ces valeurs dans le développement (1) l'on obtient la série cherchée

$$
1=\frac{1}{\pi} \sum_{n=1}^{n=\infty} \frac{\operatorname{Sin} \frac{n \pi}{4}}{n 2^{\frac{n}{2}}}\left[\frac{1}{(1-x)^{n}}+\frac{1}{(1+i x)^{n}}+\frac{1}{(1+x)^{n}}+\frac{1}{(1-i x)^{n}}\right]
$$

Cette égalité (2) a lieu pour tous les points $x$ situés dans l'aire $A B C D$. La série qui forme le second membre est encore convergente dans l'espace indéfini situé en dehors des quatre cercles; mais sa somme est alor's nulle.

Dans le cas actuel il serait aisé de sommer directement la série (2). lin effet remarquant que

$$
\operatorname{Sin} \frac{n \pi}{4}=\frac{e^{\frac{n \pi i}{4}}-e^{-\frac{n \pi i}{4}}}{2 i}
$$

et que la série

$$
u+\frac{u^{2}}{2}+\ldots+\frac{u^{n}}{n}+\ldots
$$


a pour somme la valeur de $-\log (1-u)$ qui s'annule avec $u$, l'on voit que la série

$$
\frac{1}{\pi} \sum_{1}^{\infty} \frac{\operatorname{Sin} \frac{n \pi}{4}}{n 2^{\frac{n}{2}}} \frac{1}{(1-x)^{n}}
$$

a pour somrne la valeur de

$$
\frac{1}{2 \pi i} \log \left(\frac{1+i-2 x}{1-i-2 x}\right)
$$

qui s'annule pour $x=\infty$. Cette fonction (3') est holomorphe à l'extérieur du cercle de centre 1 et de rayon $\frac{1}{\sqrt{2}}$; elle est représentée par la série $(3)$ à l'extérieur de ce cercle. Au point $x=0$ la fonction $\left(3^{\prime}\right)$ et, par suite, la fonction (3) prennent la valeur $\frac{1}{4}$.

Faisant la même remarque pour chacune des séries particlles qui constituent la série (2), l'on voit que cette série a pour somme

(4) $\frac{1}{2 \pi i}\left[\log \frac{1+i-2 x}{1-i-2 x}+\log \frac{1+i-2 x i}{1-i-2 x i}+\log \frac{1+i+2 x}{1-i+2 x}+\log \frac{1+i+2 x i}{1-i+2 x i}\right]$ où il faut prendre pour chacun des logarithmes la détermination qui s'annule à l'infini. Ces quatre logarithmes sont des fonctions holomorphes de $x$, le premier à l'extérieur du cercle de centre +1 , le second à l'extérieur du cercle de centre $+i$, le troisième du cercle de centre -1 , le quatrième du cercle de centre $-i$; de plus chacun de ces logarithmes prend pour $x=0$ la valeur $\frac{\pi i}{4}$. Or la fonction (4) peut s'écrire

$$
\frac{1}{2 \pi i} \log \frac{(1+i-2 x)(1+i-2 x i)(1+i+2 x)(1+i+2 x i)}{(1-i-2 x)(1-i-2 x i)(1-i+2 x)(1-i+2 x i)} ;
$$

d'après ce qui précède cette fonction (5) est holomorphe dans l'aire indéfinie située à l'extérieur des quatre cercles; maiș elle est constante dans cette aire car elle se réduit ̀̀ $\frac{1}{2 \pi i} \log 1$; donc elle est nulle dans cette aire indéfinie puisqu'elle est nulle à l'infini. Cette mème fonction (5) est holomorphe dans l'aire finie $A B C D$; mais elle est constante dans cette aire, car elle est égale à $\frac{1}{2 \pi i} \log 1$; elle est donc dans l’aire $A B C D$ 
égale à l'unité puisqu'elle est égale à l'unité pour $x=0$ comme nous l'avons vu.

\section{Exemple II.}

Pour indiquer un exemple dans lequel l'un des cercles limites tourne sa concavité vers l'intérieur de l'aire, considérons la surface située

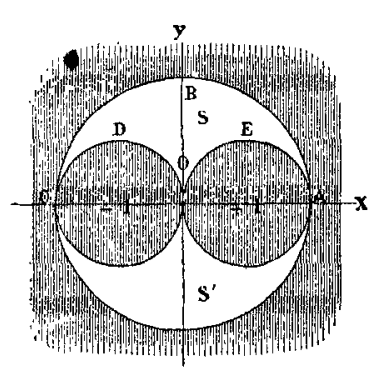

(6) à l'intérieur du cercle de centre 0 et de rayon 2 et à l'extérieur des cercles de centres -1 et +1 et de rayon 1. Cette surface se compose de deux parties séparées $S$ et $S^{\prime}$. Nous allons former une série de fractions rationnelles représentant l'unité dans l'aire $S$ et zéro dans $S^{\prime}$.

Soit $x$ un point de l'aire $S$, on a

$$
1=\frac{1}{2 \pi i} \int \frac{d z}{z-x}
$$

l'intégration étant faite sur le contour de $S$

\section{$A B C D O E A$.}

En partageant l'intégrale en trois parties relatives aux trois demi-cercles qui limitent l'aire $S$, on a

$$
1=\frac{1}{2 \pi i} \int_{A B C} \frac{d z}{z-x}+\frac{1}{2 \pi i} \int_{C D O} \frac{d z}{z-x}+\frac{1}{2 \pi i} \int_{O E A} \frac{d z}{z-x} .
$$

Dans la première intégrale mod. $x<$ inod. $z ;$ donc

$$
\frac{1}{z-x}=\frac{1}{z}+\frac{x}{z^{2}}+\ldots+\frac{x^{n}}{z^{n+1}}+\ldots
$$

dans la seconde mod. $(x+1)>\bmod \cdot(z+1)$

$$
\frac{1}{z-x}=-\frac{1}{(x+1)}-\frac{z+1}{(x+1)^{2}}-\ldots-\frac{(z+1)^{n-1}}{(x+1)^{n}}-\ldots
$$

enfin dans la troisième

$$
\frac{1}{z-x}=-\frac{1}{x-1}-\frac{z-1}{(x-1)^{2}}-\ldots-\frac{(z-1)^{n-1}}{(x-1)^{n}}-\ldots
$$


Portant dans l'équation (6) et développant on a

$$
1=\frac{1}{2 \pi i}\left[A_{0}^{(0)}+\sum_{n=1}^{n=\infty}\left(A_{n}^{(0)} x^{n}+\frac{A_{n}^{(-1)}}{(x+1)^{n}}+\frac{A_{n}^{(1)}}{(x-1)^{n}}\right)\right] .
$$

Les valeurs des coefficients sont données par les formules suivantes:

$$
\begin{gathered}
A_{0}^{(0)}=\int_{A B C} \frac{d z}{z}=\pi i \\
A_{n}^{(0)}=\int_{A B C} \frac{d z}{z^{n+1}}=\frac{1}{2^{n}} \int_{0}^{\pi} e^{-n \theta i} i d \theta=\frac{1}{n 2^{n}}\left[1-(-1)^{n}\right] \\
A_{n}^{(-1)}=-\int_{D O}(z+1)^{n-1} d z=-\int_{\pi}^{\delta} e^{n \theta i} i d \theta=-\frac{1}{n}\left[1-(-1)^{n}\right] \\
A_{n}^{(1)}=-\int_{O E A}(z-1)^{n-1} d z=-\int_{\pi}^{0} e^{n \theta i} i d \theta=-\frac{1}{n}\left[1-(-1)^{n}\right] .
\end{gathered}
$$

L'on a donc le développernent cherché

$$
1=\frac{1}{2}+\frac{1}{2 \pi i} \sum_{n=1}^{n=\infty} \frac{1-(-1)^{n}}{n}\left[\frac{x^{n}}{2^{n}}-\frac{1}{(x+1)^{n}}-\frac{1}{(x-1)^{n}}\right]
$$

La série qui forme le second membre converge encore dans l'aire $S^{\prime}$ mais sa somme est alors nulle.

L'on peut encore ici se rendre compte a priori des propriétés de la série (8). Soit posé

$$
\varphi(x)=\sum_{n=1}^{n=\infty} \frac{1-(-1)^{n}}{n}\left[\frac{x^{n}}{2^{n}}-\frac{1}{(x+1)^{n}}-\frac{1}{(x-1)^{n}}\right]
$$

Cette fonction existe dans les aires $S$ et $S^{\prime}$. La série partielle

$$
\sum_{1}^{\infty} \frac{1-(-1)^{n}}{n} \cdot \frac{x^{n}}{2^{n}}
$$

définit une fonction holomorphe dans le cercle de centre 0 et de rayon 2 ; cette fonction est la détermination de 
qui s'annule pour $x=0$. La série partielle

$$
-\sum_{1}^{\infty} \frac{1-(-1)^{n}}{n} \cdot \frac{1}{(x+1)^{n}}
$$

définit une fonction holomorphe à l'extérieur du cercle de centre -1 et de rayon 1 ; c'est la détermination de

$$
\log \frac{x}{x+2}
$$

qui s'annule à l'infini. Enfin la troisième série partielle est, dans tout l'espace situé en dehors du cercle de centre 1 et de rayon 1 , égale à la détermination de

$$
\log \frac{x-2}{x}
$$

qui s'annule à l'infini.

La fonction $\varphi(x)$ est donc holomorphe dans les deux aires $S$ et $S^{\prime}$ et elle est égale dans ces aires $\grave{a}$

$$
\varphi(x)=\log \frac{2+x}{2-x}+\log \frac{x}{x+2}+\log \frac{x-2}{x}
$$

les logarithmes ayant les déterminations indiquées. Mais on voit immédiatement que le second membre est indépendant de $x$ et égal à $\log (-1)$. Donc $\varphi(x)$ est constant dans les aires $S$ et $S^{\prime}$. Pour avoir la valeur constante de $\varphi(x)$ dans $S$ il suffit de prendre la valeur en un point de $S$, par exemple au point $x=\varepsilon i, \varepsilon$ étant positif et infiniment petit. Alors $\log \frac{2+\varepsilon i}{2-\varepsilon i}$ est infiniment petit; quant à la somme $\log \frac{x}{x+2}+\log \frac{x-2}{x}$, elle est égale à la valeur que prend au point $\varepsilon i$ la détermination de $\log \frac{x-2}{x+2}$ qui devient nulle à l'infini, la variable $x$ ne pouvant pas traverser la droite $A O C$. Or la valeur de ce logarithme au point $s i$ est $\pi i$. Donc si $x$ est un point de l'aire $S$

$$
\varphi(x)=\pi i
$$

on voit de même que, dans l'aire $S^{\prime \prime}$, 


$$
\varphi(x)=-\pi i
$$

Cela résulte encore de ce que la série (9) qui sert de détinition à $\varphi(x)$ est une fonction impaire. La série (8) étant égale à

$$
\frac{1}{2}+\frac{1}{2 \pi i} \varphi(x)
$$

a pour somme 1 dans l'aire $S$ et 0 dans $S^{\prime}$.

Remarque I. Ces exemples ouvrent la voie à d'autres recherches sur un mode particulier d'existence des fonctions; l'on voit en effet que dans les exemples précédents l'on a composé des fonctions holomorphes avec des fonctions à déterminations multiples qui sont ici des logarithmes. Je me propose d'examiner si l'on ne peut pas obtenir des résultats analogues en remplaçant les logarithmes par d'autres fonctions à déterminations multiples comme par exemple

où $P(x)$ est un polynôme.

$$
\int \frac{d x}{\sqrt{P(x)}}
$$

Remarque II. Si nous reprenons la série du premier exemple,

$$
\psi(x)=\frac{1}{\pi} \sum_{n=1}^{n=\infty} \frac{\operatorname{Sin} \frac{n \pi}{4}}{{ }_{n} 2^{\frac{n}{2}}}\left[\frac{1}{(1-x)^{n}}+\frac{1}{(1+i x)^{n}}+\frac{1}{(1+x)^{n}}+\frac{1}{(1-i x)^{n}}\right],
$$

nous voyons que la série $\phi^{\prime}(x)$ constituée par les dérivées des termes de la série $\phi(x)$

$$
f^{\prime}(x)=\frac{1}{\pi} \sum_{n=1}^{n=\infty} \frac{\sin \frac{n \pi}{4}}{2^{\frac{n}{2}}}\left[\frac{1}{(1-x)^{n+1}}-\frac{i}{(1+i x)^{n+1}}-\frac{1}{(1+x)^{n+1}}+\frac{i}{(1-i x)^{n+1}}\right]
$$

est convergente aux mêmes points que la série $\psi(x)$ et a constamment pour somme zéro. On le vérifie facilement, car si l'on remplace $\sin \frac{n \pi}{4}$ par $\frac{1}{2 i}\left(e^{\frac{n \pi i}{4}}-e^{-\frac{n \pi i}{4}}\right)$, l'on n'a plus qu'à sommer des progressions géométriques.

D'une façon générale, la série $\phi^{(k)}(x)$ constituce par les dérivées d'ordre $k$ des termes de la série $\phi(x)$ est convergente aux mêmes points que cette série $\phi(x)$ et a constamment pour soinme zéro. 
Soit alors $F(x)$ une série de fractions rationnelles convergente dans les mêmes régions que $\psi(x)$; la série

$$
F(x)+\lambda_{1} \psi^{\prime}(x)+\lambda_{2} \psi^{\prime \prime}(x)+\ldots+\lambda_{k} \psi^{(k)}(x)
$$

(où $\lambda_{1}, \lambda_{2}, \ldots \lambda_{k}$ sont arbitraires) est une série de fractions rationnelles possédant les mêmes régions de convergence et la même somine que $F(x)$. Par exemple la série

$$
\psi^{\prime \prime}(x)+\lambda_{1} \psi^{\prime}(x)+\lambda_{2} \psi^{\prime \prime}(x)+\ldots+\lambda_{k} \psi^{\prime(k)}(x)
$$

possède les mêmes propriétés que $\psi(x)$.

Les remarques précédentes conduisent ì une autre conséquence. Considérons, par exemple, la série

$$
S(x)=\frac{1}{x^{2}}+\lambda \psi^{\prime}(x)
$$

où $\lambda$ désigne une constante; cette série $S(x)$ est convergente aux mềmes points que $\psi(x)$ et représente une même fonction analytique $\frac{1}{x^{2}}$ dans toutes ses régions de convergence. Intégrons cette série terme à terme, nous aurons une autre série de fractions rationnelles

$$
S_{1}(x)=-\frac{1}{x}+\lambda \psi(x)+C
$$

convergente aux mêmes points que $S(x)$, mais représentant dans les deux régions de convergence de $S(x)$ deux fonctions analytiques différant par une constante arbitraive $\lambda$. En effet $S_{1}(x)=-\frac{1}{x}+C$ dans l'espace indéfini extérieur aux quatre cercles; et $S_{1}(x)=-\frac{1}{x}+\lambda+C$ dans l'aire finie $A B C D$ qui comprend l'origine.

Donc, si une série de fractions rationnelles représente une même fonction analytique dans toutes ses régions de convergence et si la série obtenue en intégrant la proposée terme à terme est cncore une série de fractions rationnelles, il peut arriver que cette série intégrale converge aux mêmes points que la proposée et représenie dans les différentes régions de convergence des fonctions analytiques différant par des constantes. 